\title{
BMJ Open The effects of alcohol pricing policies on consumption, health, social and economic outcomes, and health inequality in Australia: a protocol of an epidemiological modelling study
}

\author{
Heng Jiang, ${ }^{\oplus 1,2}$ Robin Room, ${ }^{1,3}$ Michael Livingston, ${ }^{1}$ Sarah Callinan, ${ }^{1}$ \\ Alan Brennan, ${ }^{4}$ Christopher Doran, ${ }^{5}$ Michael Thorn ${ }^{6}$
}

To cite: Jiang $\mathrm{H}$, Room $\mathrm{R}$, Livingston $\mathrm{M}$, et al. The effects of alcohol pricing policies on consumption, health, social and economic outcomes, and health inequality in Australia: a protocol of an epidemiological modelling study. BMJ Open 2019;9:e029918. doi:10.1136/ bmjopen-2019-029918

- Prepublication history for this paper is available online. To view these files, please visit the journal online (http://dx.doi org/10.1136/bmjopen-2019029918).

Received 25 February 2019 Revised 16 April 2019 Accepted 15 May 2019
Check for updates

(C) Author(s) (or their employer(s)) 2019. Re-use permitted under CC BY-NC. No commercial re-use. See rights and permissions. Published by BMJ.

For numbered affiliations see end of article.

Correspondence to

Dr Heng Jiang;

jason.jiang@latrobe.edu.au

\section{ABSTRACT}

Introduction Alcohol use and misuse are associated with substantial health and social issues in Australia and internationally. Pricing policy is considered as one of the most effective means to reduce risky drinking and related harms. This protocol paper describes a study that will model and estimate the effects, effectiveness and costbenefit of alcohol pricing policy initiatives in reducing risky drinking, health and social harms, and health inequalities among subpopulations in Australia.

Methods and analysis The study is a modelling and epidemiological study using data from various resources, such as survey, previous literatures and response agencies. A number of statistical procedures will be undertaken to evaluate the impact of different alcohol pricing policy initiatives on various outcomes, including alcohol consumption in population subgroups, and health and social problems, and to measure health inequalities and cost-effectiveness of those proposed pricing policies, such as a $10 \%$ tax increase on all alcohol beverages or introduction of a minimum unit price.

Ethics and dissemination The ethics approval of this study was obtained from the College Human Ethics SubCommittee of the La Trobe University on 9 November 2017 (Ref: S17-206). While examining the heterogeneous effects of price policy across population subgroups, this study will provide the first comprehensive estimates of the likely impacts of alcohol price changes on health inequalities. The study will also provide sophisticated economic analyses of the impact of price policy changes, which is critical information for policy makers and will assist policy makers in directing resources to a more efficient alcohol strategy. Results will be made available to communities and societies, health departments and other researchers.

\section{INTRODUCTION}

Alcohol use and misuse are an important public health policy issue because of their associations with acute injuries and chronic diseases. ${ }^{1}$ Price-based interventions, predominantly through changes to taxation, have been shown to be one of the most effective means
Strengths and limitations of this study

- Using both survey and response agency data, this is the first study to model and estimate the effects, effectiveness and cost-benefit of alcohol pricing policy initiatives in reducing risky drinking, health-related harms and health inequalities in Australia.

- Using Australian Harm to Others Survey and National Drug Strategy Household Survey data and data from systematic reviews, dose-response relationships between drinkers' alcohol consumption and various social harms will be measured. This is the first study that examines the effects of alcohol pricing policy initiatives on various social outcomes in the field.

- The protocol will serve as a guideline for other countries to build up a systematic modelling approach to model and estimate the effects of alcohol pricing policy on non-communicable diseases, injuries, assaults, violence, homicide and harm to others among subpopulations.

- Recall bias in the survey interview may mean our results on alcohol consumption or purchasing are underestimated.

to reduce the level of alcohol consumption and of related health and social problems. ${ }^{23}$ Research suggests that increasing alcohol tax or price can lead to reductions in consumption, fatal traffic accidents, deaths from liver cirrhosis, workplace injuries, violence and other crimes. ${ }^{4}$

Although the average level of alcohol consumption in Australia has declined somewhat in recent years, several alcohol-related harms have steadily increased. ${ }^{5}$ This may reflect heterogeneity in consumption trends, with the reductions in the general population not necessarily reflected among heavy drinkers. Previous research has argued that understanding how policies affect different classes of drinkers is key to understanding 
the likely effects of policy on harm. ${ }^{6}$ Our previous work has shown that the heaviest $20 \%$ of Australian drinkers drank over $80 \%$ of all alcohol consumed in the last year, ${ }^{7}$ and a high proportion of the purchases of these heavy drinkers was of low-price alcohol. ${ }^{8}$ The alcohol taxation system is complex in Australia, with a combination of both volumetric and ad valorem taxes, varying in their application by the type of alcohol product and by the range of alcohol content. ${ }^{9}$ For example, volumetric excise taxes are levied on beer and spirits (ie, tax is based on the volume of alcohol contained in the product), while ad valorem excise taxes (ie, based on the value of the product) are applied on wine. Off-premise purchases are generally a cheaper source of alcohol than on-premise drinks and make up about $80 \%$ of the alcohol market in Australia. ${ }^{10}$ Cheapest by far, because of the low taxes, is off-premise cask wine; a $4 \mathrm{~L}$ wine cask, containing $420 \mathrm{~g}$ of alcohol, may sell for as little as $\$ 13$ (sales tax was $\$ 3.77$ in 2018), while the same amount of alcohol in two non-premium $700 \mathrm{~mL}$ spirit bottles with $37 \%$ alcohol volume sells for $\$ 70$ (volumetric tax was $\$ 43.43$ in 2018). ${ }^{11}$ Our previous analyses revealed that both drinkers who are socioeconomically disadvantaged and risky drinkers are more likely to purchase cheap alcohol and to experience more alcohol-related harms than other drinkers. ${ }^{812}$

In Australia in the last decade, the rate of risky drinking among young people has been decreasing, while risky drinking among older adults has been increasing; the latter trend is associated with more diseases, injuries and hospitalisations among older age groups. ${ }^{13}$ (Short-term risky drinking was defined by the National Health and Medical Research Council in 2009 as drinking more than four Australian standard drinks (ASDs, $10 \mathrm{~g}$ ethanol) in an occasion at least once a month, and long-term (lifetime) risk was defined as drinking on average more than two ASDs per day or 14 ASDs per week). ${ }^{14}$ Our preliminary analyses found that $48 \%$ of older Australian heavy drinkers (aged 55 or over) drank low-price alcohol $(<\$ 1$ per ASD, containing $10 \mathrm{~g}$ of ethanol) in the last 12 months. ${ }^{12}$ Furthermore, the evidence of pricing policy impact on young heavy drinkers is not clear. ${ }^{15}$ Disregarding the differential effectiveness of pricing policies in reducing alcohol consumption across different demographic groups (eg, age groups) remains a weakness of the existing literature, meaning that current estimates of the effects of alcohol prices on alcohol-related harms are flawed.

Recent policy debates about setting a minimum price or raising taxes on alcohol as a public health prevention strategy ${ }^{16}$ have posed the question as to whether any increase in the alcohol price or tax would disproportionately affect moderate drinkers. A recent parliamentary inquiry into alcohol-related harms has recommended reforms to understand the effectiveness of taxation policies in reducing the social costs of alcohol. ${ }^{17}$ Thus, Australian policy makers need more evidence on the impacts of different pricing initiatives on alcohol consumption among different subpopulations and on social and health outcomes.

Studies in the UK have estimated the effects of different alcohol price initiatives on consumption and health outcomes $^{1819}$ and found that lower income and more hazardous drinkers are more price responsive than higher income and moderate drinkers. There has been no such study in Australia using national representative survey data. Two recent studies in Australia were conducted based on scanned samples of off-premise purchases in Victoria, or on national aggregate data, ${ }^{9}{ }^{20} 21$ but one covered only a small part of alcohol purchasing, while the second was not able to provide any estimates for population subgroups. Thus, the effects of changes in price policies on consumption of on-premise and off-premise alcoholic beverages among different subpopulations remain unclear. Some previous Australian studies have attempted to model the likely impacts of alcohol pricing policies on health outcomes, ${ }^{92}$ but they have relied on price elasticities from the UK or meta-analyses and generally have not examined the differential effects of pricing across subpopulations-an issue that was identified as critically important in a recent Lancet paper. ${ }^{19}$ Our recent estimation of price elasticity of demand for 11 beverage categories, based on Australian survey data, ${ }^{23}$ can fill this research gap and provide more reliable estimates of the effects of price policy initiatives on alcohol consumption among different subpopulations.

Previous Australian research ${ }^{24}$ has demonstrated that the negative impacts associated with alcohol consumption extend well beyond health. These impacts include crime, lost productivity, damage to property, reduced well-being and many more. Most studies examining policy impacts have been restricted to health impacts, meaning that the overall impact of policy change has likely been underestimated. This study will develop innovative estimates of the impact of changes in consumption on a number of these social harms, allowing for broader estimates of the overall impact of pricing policies.

An important concern that has been raised in the literature about higher rates of alcohol taxation is the potential impact on socioeconomic disadvantaged groups. ${ }^{23}$ Alcohol taxation is considered regressive, suggesting that those that can least afford alcohol will suffer the most. However, in terms of health inequalities, this may be regarded as a positive effect, with greater health gains from increased alcohol prices likely among disadvantaged groups. ${ }^{25} 26$ There is promising evidence from the UK and the USA that alcohol pricing policies indeed have the potential to reduce socioeconomic health inequalities, ${ }^{27}{ }^{28}$ which are known to be significantly influenced by alcohol-related harms, ${ }^{29}$ but there have been no Australian studies on this topic. Furthermore, the international literature currently focuses only on the impact of pricing policies on alcohol consumption and negative health outcomes. This study will be the first to examine the potential role of alcohol pricing policies in reducing health inequalities. 
Previous Australian economic analyses on alcohol policies have relied on aggregate level data, ${ }^{022}$ have neglected subpopulation groups and have not considered the wider range of social harms due to alcohol consumption. This project will fill this void and identify the most economic efficient pricing interventions to reduce alcohol-related health and social harms in vulnerable populations, such as risky drinkers, drinkers with low socioeconomic status (SES), and young or older risky drinkers.

\section{Objectives}

The objectives of the project are to

1. Estimate the impact of a series of plausible pricing policies on alcohol consumption across different subgroups of the population (eg, age, income and drinking levels) based on price elasticities developed by the project team.

2. Conduct a comprehensive systematic review of systematic reviews to better understand the dose-response relationships between alcohol consumption and health and social harms.

3. Estimate the impacts of alcohol pricing policies on health inequalities.

4. Estimate the impacts of alcohol pricing policies on economic outcomes via economic evaluation techniques.

\section{METHODS AND ANALYSIS}

The study is an economic modelling and epidemiological study using survey data (ie, the Australian surveys of the International Alcohol Control Study survey and of the Australian Alcohol's Harm to Others (AHTO) survey), data from published articles and reports, and secondary data from a number of agencies, such as the Australian Bureau of Statistics (ABS) and the Australian Institute of Health and Welfare (AIHW). Based on a review of existing Australian policy documents and studies, ${ }^{89}$ eight pricing policy initiatives are proposed and will be modelled in this study, including five alcohol tax and three minimum unit pricing policies (box 1). Furthermore, a broad range of scenarios, including policies targeted at particular

\section{Box 1 Proposed pricing policy initiatives}

\section{Tax policy initiatives}

1. Increase excise rate by $10 \%$ for all off-premise beverages.

2. Replace the wine equalisation tax with a volumetric excise rate equal to the current excise tax rate applicable to spirits and RTDs.

3. Apply a uniform excise tax rate to all beverages equal to the current excise tax rate applicable to spirits and RTDs.

4. Apply a uniform excise tax rate to all beverages equal to a $10 \%$ increase in the current excise tax rate applicable to spirits and RTDs.

5. Apply a uniform excise tax rate to all beverages equal to a $20 \%$ increase in the current excise tax rate applicable to spirits and RTDs.

\section{Minimum unit pricing policy initiatives}

6. Introduce a floor price on all beverage categories at $\$ 1.00$ per ASD.

7. Introduce a floor price on all beverage categories at $\$ 1.30$ per ASD.

8. Introduce a floor price on all beverage categories at \$1.50 per ASD. beverages (eg, low-cost wine), can be simulated using this model. We will engage in stakeholder engagement to define the priorities for further specific modelling. The study procedure is elaborated below in subsections corresponding to each project objective.

The project will be completed within 3years, starting from March 2018. Stata V.14 ${ }^{30}$ will be used to analyse survey data, and MS Excel will be used to calculate relative risks (RRs) for and effects of pricing policy change on various health and social outcomes.

\section{Patient and public involvement}

Patients and the public were not involved in the development of the research design, analysis or interpretation of the study. The authors did not conduct any interaction or intervention with individuals about whom data were obtained.

\section{Estimating the impact of pricing policy change on alcohol consumption among subpopulations}

In this project, alcohol consumption, purchasing and price data were collected from the Australian International Alcohol Control (IAC) 2013 survey-a national representative telephone survey collecting data on the experience of alcohol consumption and purchasing from 2020 Australians (age 16+) across Australia who spoke English. The computer-assisted telephone interview was reached by random digit dialling to landlines $(60 \%)$ or mobile phones (40\%). People who drank more than five ASDs on an occasion monthly or more were oversampled. After weighting to compensate for the oversampling, the sample was generally representative of the Australian adult population with a cooperation rate of $51.5 \%$ and an American Association for Public Opinion Research (AAPOR) response rate 3 of $37.2 \%$. Details of the survey method, questionnaire and technical report can be found in Jiang $e t a \vec{l}^{31}$ and in Livingston and Callinan. ${ }^{32}$

The changes in mean consumption on 11 beverage categories (eg, on-premise and off-premise full-strength beer, low-strength to middle-strength beer, bottle wine, spirits, ready-to-drinks and off-premise cask wine) after a change in price can be estimated based on the price elasticities derived from our econometric modelling. ${ }^{23}$ Elasticities are estimated for subpopulation groups based on drinking level (moderate, hazardous and harmful) or income (three levels), split by three age groups and two gender groups. The three drinking levels are defined as moderate drinkers ( $\leq 14$ ASDs per week), hazardous drinkers (14-42 ASDs for men and 14-35 ASDs for women) and harmful drinkers (>42ASDs for men and $>35$ ASDs for women). The three income groups were split into fairly equal observations based on the annual income in the respondent's household: lower income $(<\$ 61000)$, middle income (\$61 000-114000) and higher income $(>11000)$. The smallest sample size in subpopulation groups is larger than 75 , sufficient for estimation within each subsample. 
The SES of each drinker can be identified by linking IAC respondent postcode information with the postcode identifiable Socio-Economic Index for Areas (SEIFA), which ranks areas in Australia according to relative socioeconomic advantage and disadvantage. ${ }^{33}$ These data enable beverage-specific consumption patterns to be modelled by level of disadvantage.

Based on the elasticity matrix presented in our previous study, ${ }^{23}$ the impact of the change in taxes or prices caused by a given policy change on consumption of different on-trade and off-trade alcoholic beverages can be estimated for each modelled subpopulation group. The formula is shown below:

$$
\% \Delta D_{i}=\left(1+E_{i, i} \% \Delta P_{i}\right)\left(1+\sum_{j \neq i}^{\forall j} E_{i, j} \% \Delta P_{j}\right)-1
$$

where $\% \Delta D_{i}$ is the estimated percentage change in alcohol demand or consumption for beverage $i, E_{i, i}$ is the own-price elasticity for beverage $i, \% \Delta P_{i}$ is the percentage change in price for beverage $i, E_{i, j}$ is the cross-price elasticity for the consumption of beverage $i$ due to a change in the price of beverage $j$, and $\% \Delta P_{j}$ is the percentage change in price for beverage $j$.

Using alcohol consumption, purchasing and price data from the Australian IAC study, we will compute the price distribution of each on-trade and off-trade beverage, which allows us to evaluate the proportion of alcohol within each beverage category sold below proposed floor prices. Descriptive analyses of the impacts of introducing different floor prices (eg, $\$ 1.00$ and $\$ 1.25$ on alcohol consumption among different drinking levels and income groups) were done in our previous report. ${ }^{8} \mathrm{~A}$ more comprehensive analysis will be done in this project, allowing us to understand how a floor price would affect consumption of different alcoholic beverages, as well as among moderate, hazardous, harmful, low-income, middle-income and high-income drinkers, split by three age groups and two gender groups. The results will be compared with other price policy scenarios in order to identify the most effective price policy to reduce alcohol-related harm.

\section{Estimating the impact of alcohol consumption change due to pricing policy on health outcomes}

An epidemiological approach will be used to model the relationship between consumption and harm, relating changes in the level of alcohol consumption to changes in prevalence of the risk of experiencing harmful health outcomes. Using this approach, the policy impact for the large number of health conditions for which there is evidence that alcohol plays a contributory role can be captured.

A search will be undertaken of the WHO International Statistical Classification of Diseases and Related Health Problems, 10th Revision (ICD-10) databank, ${ }^{34}$ using the term 'alcohol', to identify disease and injury categories attributable to alcohol. All identified alcohol-related health conditions will be split into four groups, including (1) wholly attributable chronic (an up-to-date list of fully alcohol-attributable chronic diseases can be found in Rehm $e t a l^{35}$ ), (2) wholly attributable acute, (3) partially attributable chronic diseases and (4) partially attributable acute.

A systematic review will then be conducted using relevant search engines, including AMED, Embase, Health and Psychosocial Instruments, PsycINFO, PubMed, Google Scholar and Web of Science to identify systematic reviews and/or meta-analyses published in English, with no geographical restrictions. Key words will be different alcohol categories and the respective outcome category, along with either 'systematic review' or 'meta-analysis'. All databases will be searched from January 2006 to identify RR of diseases and injuries due to alcohol consumption, which have been studied in the last 10 years.

After the systematic review, the most up-to-date RR relationship with alcohol consumption of each disease and injury category will be obtained, and alcohol attributable fractions (AAFs) for mortality and morbidity will be calculated. AAFs can be interpreted as the proportion of an outcome in a specific population that would not have occurred if there had been no alcohol use. ${ }^{36} \mathrm{AAFs}$ can be calculated for multiple levels of alcohol consumption as follows:

$$
A A F=\frac{\sum_{x=1}^{n} P(x)(R R(x)-1)}{\sum_{x=0}^{n} P(x)(R R(x)-1)+1}
$$

where $P(x)$ is the prevalence of current drinkers consuming $x$ grams of alcohol daily (calculated by the IAC survey of alcohol consumption distributions across age and gender groups). $R R(x)$ is the $\mathrm{RR}$ of diseases or injuries for drinkers drinking $x$ grams of alcohol daily compared with abstainers, where $x=0$ represents non-drinking. The maximum daily consumption is capped at $300 \mathrm{~g}$ /day (30 ASDs). As an example, the RR estimates for malignant neoplasms of the lip, oral cavity and pharynx can be collected from a previous meta-analysis (eg, Bagnardi $e t$ $\left.a l^{37}\right)$ - they are 1.00 for abstainers (reference group), 1.13 for drinking one to two ASDs per day; 1.83 for drinking three to five ASDs per day and 5.31 for drinking over five ASDs per day. Using the prevalence of drinking among men aged 16-24 across different drinking levels in the IAC survey, the AAF for malignant neoplasm of the lip, oral cavity and pharynx in men aged 16-24 who drank alcohol moderately between one and two ASDs per day can be calculated as $[0.495 *(1.13-1)] /[0.107 *(1.00-1)+$ $(0.495 *(1.13-1)+0.214 *(1.83-1)+0.184 *(5.31-1)+1]=3.2$ $\%$.

Potential beneficial effects of alcohol consumption will also be calculated by means similar to those used for negatively affected chronic conditions, with the maximum daily consumption set at $50 \mathrm{~g} /$ day. The beneficial effects of alcohol consumption were found mainly associated with light or moderate alcohol consumption, 
which means drinking no more than five standard drinks $(50 \mathrm{~g})$ per day, which may reduce risks for some cardiovascular diseases and diabetes. ${ }^{38} 39$

The links between alcohol consumption and risk of chronic harms are affected by 'time lags'. Previous systematic reviews and burden of disease studies have suggested that the full effects of changes in consumption on health (including cancers) can vary between 1 and 20 years. ${ }^{40}$ This study will use a lag of 10 years to estimate the 'full effect' on most of chronic diseases in our model, and a lag of 20 years will be used to estimate the effect of drinking on cancer diseases.

The majority of results of chronic health conditions will be presented for the 10th year following policy implementation and the 20th year for cancer diseases only, with a linear progression to full effect on risk. Previous studies have not considered these lagged effects.

Australian mortality data are compiled in the National Causes of Death Database maintained by the ABS. Alcohol-related causes of death data from 2016 to 2017 will be requested from ABS, aggregated by age group, gender, socioeconomic index and ICD-10 conditions. Australian hospital admissions can be collected from the National Hospital Morbidity Database (NHMD), archived by AIHW. The NHMD includes patient admission records in all public and private hospitals (acute or psychiatric), and private free-standing day hospital facilities. The project team will collect all hospital statistical separations in Australia admitted from 2016 to 2017.

Based on the calculated AAFs and Australian mortality and morbidity data, the health effects of the alcohol consumption changes estimated in the first stage of the analyses will be estimated for each proposed price intervention. Health effects will be evaluated in terms of deaths, hospitalisations and the standard WHO measure, the disability-adjusted life year (DALY).

\section{Estimating the impact of alcohol consumption change due to pricing policy on social outcomes}

This study will innovatively estimate the effects of different alcohol pricing policy change on social outcomes in Australia, such as effects on reducing domestic violence, child abuse, time of caring for drinkers, crime, alcohol and other drug treatments, health service usage, and work absence due to drinking in Australia. The second systematic review in the project will be conducted to identify the relationship between alcohol consumption and social harms (particularly focusing on alcohol-related assaults, violence, aggressions, crime and work absence). For the present purposes, these social outcomes are assumed to be a consequence of acute drinking rather than average or long-term drinking with lagged effects. Using the same databases, systematic reviews or meta-analyses on those social harms will be initially conducted. If there is no existing systematic review for some social harms, then we will systematically review all published observational studies and grey literature studies to identify the related dose-response relationships, such as a dose- response relationship between alcohol consumption and work absence.

Risk ratios will be estimated for social harms where previously published estimates are identified and will be applied to Australian sources of data to provide estimates of the impact of price policies on social harms. For example, the most recent estimates of the AAFs on crime and workplace harm among subpopulations can be derived from a study conducted by the Sheffield Alcohol Research Group in the UK. ${ }^{41}$ We will apply these estimates to the relevant Australian data (eg, the detailed national crime data compiled by the $\mathrm{ABS}^{42}$ ) to estimate policy effects. It is expected that limited existing evidence will be available to develop these risk ratios, and they are likely to vary between societies, so we are also proposing analyses of survey data to derive new estimates of the likely impact of changes in drinking on social harms. We will collect data from the Australian AHTO survey 2008, ${ }^{24}$ a proposed second wave of AHTO survey in 2019 and the National Drug Strategy Household Survey 2016, ${ }^{43}$ and we will analyse relationships between domestic violence, child abuse, caring time, police and services usage, and the drinking level of the drinker who caused these harms.

\section{Estimating the impact of price policy change on health inequality}

In this analysis, two separate approaches use different measures of socioeconomic position. First, the survey population is divided into three income groups based on respondents' household income. This approach reflects that household income (rather than the SES of the respondent's neighbourhood) is considered a key driver of alcohol purchasing. However, some studies suggest that the socioeconomic group may drive health inequalities more than income. ${ }^{28}$ Therefore, the neighbourhood-based SES (identified by the ABS postal SEIFA index) of each drinker will also be used from the Australian IAC data. The SEIFA index was developed by the ABS to rank areas in Australia according to relative socioeconomic advantages and disadvantages, ${ }^{33}$ and the 2016 SEIFA index will be used in this study (more information about the SEIFA index was provided in the SEIFA 2016 technical paper ${ }^{44}$ ). Using these two approaches, an estimate will be made of the impacts of each of our proposed alcohol price policy initiatives on inequalities in hospitalisations, deaths and DALYs.

\section{Estimating the impact of price policy change on economic outcomes}

The impact of alcohol consumption change due to different price policy changes on economic outcomes will be evaluated using both cost-effectiveness analysis (CEA) and cost-benefit analysis (CBA).

CEA will adopt standardised methods, as used by the authors in previous studies. ${ }^{24546}$ The methods are international best practice and include the adoption of a societal perspective; transparent and scientific methods to identify, measure and value both costs and outcomes; 
modelling and uncertainty testing of epidemiological and costing input parameters; and interpretation of results within a broader decision-making framework. The cost associated with each pricing scenario will be quantified and adjusted to account for subsequent changes in alcohol-related taxation revenue and healthcare costs. The DALY will be the health metric used in the CEA, derived from the epidemiological modelling outlined above. Consistent with WHO modelling, the analysis will model costs and outcomes for a 10-year period, discounting future costs and health outcomes at a rate of $3 \%$ per year. The costs and health outcomes will be summed to determine the incremental cost-effectiveness ratio. Monte Carlo analysis will be used to derive $95 \%$ uncertainty intervals for all outcomes and to determine the probability of intervention cost-effectiveness against a cost utility analysis threshold of $\$ 50000$ per quality-adjusted life year. The incremental cost-effectiveness ratio results are displayed on a cost-effectiveness plane with affordability issues addressed in an acceptability curve. Results will be considered in the context of strength of evidence, capacity of the intervention to reduce inequity, feasibility and sustainability.

The CBA will quantify the social benefits arising from the alcohol price policy initiatives, including savings in healthcare costs, reduced levels of crime and domestic violence, crime and productivity gains. The results of the CBA are expressed as a ratio, expressed as the number of dollars of community benefit per dollar of cost. Consistent with guidelines for undertaking an economic evaluation, an extensive sensitivity and uncertainty analysis will be conducted. For example, in order to ensure findings are robust, the empirical estimates of variations of price elasticities by beverage type, consumption level, income and place of purchase will be used to model the impacts of price change on alcohol consumption and harms compared with our results.

\section{ETHICS AND DISSEMINATION}

Ethical approval was received from the College Human Ethics Sub-Committee of La Trobe University on 9 November 2017 (Ref: S17-206). Pricing policy is considered as one of the most effective interventions to reduce alcohol-related harm. Policy debates in Australia have identified a lack of evidence regarding the extent to which price policies affect heavy drinkers and whether increased prices might penalise moderate drinkers. This research will provide key research evidence to inform current policy debate. As well as examining the heterogeneous effects of price policy across population subgroups, this project will provide the first comprehensive estimate of the likely impacts of alcohol price changes on health inequalities, which remain a major problem in Australia. ${ }^{47}$ The research will also provide first estimates of the effects of price policy interventions on social outcomes, in the first analysis to fully account for the time lags between consumption and some harms, developing the evidence base on which policy decisions can be made. Additionally, the proposed study will provide sophisticated CBA and CEA of the impact of price policy changes, which is critical information for policy makers.

The study will be conducted in accordance with the National Health and Medical Research Council national statement on ethical conduct in research, as well as the approved study protocol. The project findings will be presented at scholarly meetings or conferences, in research reports and in peer-review journal articles; the collaborative and intersectoral investigators will disseminate these findings via newsletters and web media in the field. The policy relevance of the study is significant, and the project team will use its strong existing connections to government and policy advocates to ensure that its findings inform policy decisions, and will ensure that the evidence developed in this study is a key consideration in any alcohol tax reform.

\section{Limitations}

Recall bias in the survey interview may affect our estimation of both consumption and prices paid, which may mean our results on alcohol consumption could be underestimates, although in aggregate, the IAC survey methodology minimises such underestimation ${ }^{32}$ and such impact on our modelling estimation should be moderate. Our sample size $(n=2020)$ allows us to conduct economic anal$y$ sis on combinations of gender $\times$ SES $\times$ drinking status or age $\times$ gender $\times$ SES or age $\times$ gender $\times$ drinking status with the smallest cell size of 30 across up to 18 subgroups. However, the sample size is not big enough for us to split the whole sample by more subgroups, such as age $\times$ drinker $\times$ income or age $\times g e n d e r \times$ drinker $\times$ income.

\section{Author affiliations}

${ }^{1}$ Centre for Alcohol Policy Research, School of Psychology and Public Health, La Trobe University, Melbourne, Victoria, Australia

${ }^{2}$ Centre for Health Equity, Melbourne School of Population and Global Health, University of Melbourne, Melbourne, Victoria, Australia

${ }^{3}$ Centre for Social Research on Alcohol and Drugs, Department of Public Health Sciences, Stockholm University, Stockholm, Sweden

${ }^{4}$ Sheffield Alcohol Research Group, School of Health and Related Research, University of Sheffield, Sheffield, UK

${ }^{5}$ Centre for Indigenous Health Equity Research, School of Health, Medicaland Applied Sciences, Central Queensland University, Brisbane, Queensland, Australia ${ }^{6}$ Foundation for Alcohol Research and Education, Canberra, Australian Capital Territory, Australia

Acknowledgements The Centre for Alcohol Policy is funded by the Foundation for Alcohol Research and Education, an independent, charitable organisation working to prevent the harmful use of alcohol in Australia (www.fare.org.au).

Contributors The study concept and design were conceived by HJ, ML, RR, SC, AB and $\mathrm{CD}$. HJ wrote the first draft of the manuscript. All authors critically reviewed the manuscript and approved the final version.

Funding This study was fully funded by a National Health and Medical Research Council Project grant (number 1141325). The datasets used in this study were from the Internal Alcohol Control Study, which was funded by the Australian National Preventive Health Agency (grant number 157R002011), and from the Australian survey on alcohol's harm to others, funded by the Foundation for Alcohol Research and Education. ML is supported by a National Health and Medical Research Council Career Development Fellowship. SC is supported by an Australian Research Council Discovery Early Career Researcher Award (DE180100016). 
Competing interests None declared.

Patient consent for publication Not required.

Provenance and peer review Not commissioned; externally peer reviewed.

Open access This is an open access article distributed in accordance with the Creative Commons Attribution Non Commercial (CC BY-NC 4.0) license, which permits others to distribute, remix, adapt, build upon this work non-commercially, and license their derivative works on different terms, provided the original work is properly cited, appropriate credit is given, any changes made indicated, and the use is non-commercial. See: http://creativecommons.org/licenses/by-nc/4.0/.

\section{REFERENCES}

1. Rehm J, Mathers C, Popova S, et al. Global burden of disease and injury and economic cost attributable to alcohol use and alcohol-use disorders. The Lancet 2009;373:2223-33.

2. Casswell S, Thamarangsi T. Alcohol and global health 3: Reducing harm from alcohol: call to action. The Lancet 2009;373:2247-57.

3. World Health Organization. Tackling NCDs: 'best buys' and other recommended interventions for the prevention and control of noncommunicable diseases. Geneva: World Health Organization, 2017.

4. Chaloupka FJ, Grossman M, Saffer H. The effects of price on alcoho consumption and alcohol-related problems. Alcohol Res Health 2002;26:22-34

5. Livingston M, Matthews S, Barratt MJ, et al. Diverging trends in alcohol consumption and alcohol-related harm in Victoria. Aust N Z J Public Health 2010;34:368-73.

6. Meier PS, Purshouse R, Brennan A. Policy options for alcohol price regulation: the importance of modelling population heterogeneity. Addiction 2010;105:383-93.

7. Livingston M. Understanding recent trends in Australian alcohol consumption. Canberra, Australia, 2016.

8. Callinan S, Room R, Livingston M, et al. Who Purchases Low-Cost Alcohol in Australia? Alcohol Alcohol 2015;50:647-53.

9. Sharma A, Vandenberg B, Hollingsworth B. Minimum pricing of alcohol versus volumetric taxation: which policy will reduce heavy consumption without adversely affecting light and moderate consumers? PLoS One 2014;9:e80936-13.

10. International E. Alcohol drinks in Australia. London: Euromonitor, 2012.

11. Current prices and products of spirits in Sepember. 2018 https:// www.danmurphys.com.au/current-offers?filters=price(25-50),variety( spirits)

12. Jiang $\mathrm{H}$, Callinan $\mathrm{S}$, Livingston $\mathrm{M}$, et al. Off-premise alcohol purchasing in Australia: Variations by age group, income level and annual amount purchased. Drug Alcohol Rev 2017;36:210-9.

13. Australian Institute of Health and Welfare. National drug strategy household survey detailed report 2013. Drug Statistic Series. Canberra: AlHW, 2015.

14. National Health and Medical Research Council. Australian guidelines to reduce health risks from drinking alcohol. Canberra: NHMRC, 2009.

15. ANPHA. Exploring the public interest case for minimum (floor) price for alcohol. In. Canberra: Australian National Preventive Health Agency 2013.

16. Chalmers J, Carragher N, Davoren S, et al. Real or perceived impediments to minimum pricing of alcohol in Australia: public opinion, the industry and the law. Int J Drug Policy 2013;24:517-23.

17. House of RepresentativesAffairs HoRSCol. Alcohol, hurting people and harming communities. Inquiry into the harmful use of alcohol in aboriginal and Torres Strait Islander communities. Canberra: The Parliament of the Commonwealth of Australia, 2015.

18. Anderson P, Chisholm D, Fuhr DC. Effectiveness and costeffectiveness of policies and programmes to reduce the harm caused by alcohol. Lancet 2009;373:2234-46.

19. Holmes J, Meng Y, Meier PS, et al. Effects of minimum unit pricing for alcohol on different income and socioeconomic groups: a modelling study. Lancet 2014;383:1655-64.

20. Doran CM, Byrnes JM, Cobiac LJ, et al. Estimated impacts of alternative Australian alcohol taxation structures on consumption, public health and government revenues. Med J Aust 2013;199:619-22.

21. Vandenberg B, Sharma A. Are Alcohol Taxation and Pricing Policies Regressive? Product-Level Effects of a Specific Tax and a Minimum Unit Price for Alcohol. Alcohol Alcohol 2016;51:493-502.
22. Doran C, Vos T, Cobiac L, et al. Identifying cost-effective interventions to reduce the burden of harm associated with alcohol misuse in Australia. Canberra: AERF, 2008.

23. Jiang $\mathrm{H}$, Livingston $\mathrm{M}$, Room R, et al. Price elasticity of on- and offpremises demand for alcoholic drinks: A Tobit analysis. Drug Alcohol Depend 2016;163:222-8.

24. Laslett $\mathrm{A}-\mathrm{M}$, Catalano $\mathrm{P}$, Chikritzhs $\mathrm{T}$, et al. The range and magnitude of alcohol's harm to others. Canberra: Alcohol Education and Rehabilitation Foundation, 2010.

25. Inequality MM. deprivation and alcohol use. Addiction 1997;92:13-20.

26. Sassi F, Belloni A, Mirelman AJ, et al. Equity impacts of price policies to promote healthy behaviours. Lancet 2018;391:2059-70.

27. Staras SA, Livingston MD, Christou AM, et al. Heterogeneous population effects of an alcohol excise tax increase on sexually transmitted infections morbidity. Addiction 2014;109:904-12.

28. Meier PS, Holmes J, Angus C, et al. Estimated Effects of Different Alcohol Taxation and Price Policies on Health Inequalities: A Mathematical Modelling Study. PLoS Med 2016;13:e1001963.

29. Probst C, Roerecke M, Behrendt S, et al. Socioeconomic differences in alcohol-attributable mortality compared with all-cause mortality: a systematic review and meta-analysis. Int $J$ Epidemiol 2014;43:1314-27.

30. StataCorp. Stata statistical software Version 14. 14th edn. College Station, Texas: Stata Press Publication, 2016.

31. Jiang $\mathrm{H}$, Callinan $\mathrm{S}$, Room R. Alcohol consumption and purchasing (ACAP) study: Survey approach, data collection procedures and measurement of the first wave of the Australian arm of the International Alcohol Control Study. In. Melbourne: Centre for Alcohol Policy Research 2014.

32. Livingston M, Callinan S. Underreporting in alcohol surveys: whose drinking is underestimated? J Stud Alcohol Drugs 2015;76:158-64.

33. Australian Bureau of Statistics. Census of Population and Housing: Socio-Economic Indexes for Areas (SEIFA), Australia, 2016 2033.0.55.001. In: Cat. 2033.0.55.001. Canberra: Australian Bureau of Statistics, 2016.

34. World health organisation. International Statistical Classification of Diseases and Related Health Problems 10th Revision, 2010.

35. Rehm J, Gmel GE, Gmel G, et al. The relationship between different dimensions of alcohol use and the burden of disease-an update. Addiction 2017;112:968-1001.

36. Jones L, Bellis MA. Updating England-specific alcohol-attributable fractions. Liverpool: Liverpool John Moores university, 2013.

37. Bagnardi V, Rota M, Botteri E, et al. Alcohol consumption and sitespecific cancer risk: a comprehensive dose-response meta-analysis. Br J Cancer 2015;112:580-93.

38. Knott C, Bell S, Britton A. Alcohol Consumption and the Risk of Type 2 Diabetes: A Systematic Review and Dose-Response Meta-analysis of More Than 1.9 Million Individuals From 38 Observational Studies. Diabetes Care 2015;38:1804-12.

39. Patra J, Taylor B, Irving H, et al. Alcohol consumption and the risk of morbidity and mortality for different stroke types--a systematic review and meta-analysis. BMC Public Health 2010;10:258.

40. Holmes J, Meier PS, Booth A, et al. The temporal relationship between per capita alcohol consumption and harm: a systematic review of time lag specifications in aggregate time series analyses. Drug Alcohol Depend 2012;123:7-14.

41. Meng Y, Sadler S, Gell L, et al. Model-based appraisal of minimum unit pricing for alcohol in Wales: An adaptation of the Sheffield Alcohol Policy Model version 3: University of Sheffield, 2014.

42. Australian Bureau of StatisticsAustralian Bureau of Statistics. 4510.0 - Recorded crime - victims, Australia, 2015. Canberra, 2016.

43. Australian Institute of Health and Welfare. National Drug Strategy Household Survey 2016: Detailed findings. Drug Statistic Series. Canberra: AlHW, 2017.

44. Australian Bureau of Statistics. Technical Paper-Socio-Economic Indexes for Areas (SEIFA) In: 2033.0.55.001. Canberra: Australian Bureau of Statistics, 2016.

45. Cobiac L, Vos T, Doran C, et al. Cost-effectiveness of interventions to prevent alcohol-related disease and injury in Australia. Addiction 2009;104:1646-55.

46. Chisholm D, Doran C, Shibuya K, et al. Comparative costeffectiveness of policy instruments for reducing the global burden of alcohol, tobacco and illicit drug use. Drug Alcohol Rev 2006;25:553-65.

47. The Senate Community Affairs Committee Secretariat. Australia's domestic response to the World Health Organization's (WHO) Commission on Social Determinants of Health report "Closing the gap within a generation". Canberra: Parliament House, 2013. 